breaking-up of the tribal organization. The detribalized native is a very difficult problem; though he may be materially more prosperous than his tribal brother, he is seldom as contented as the person who lives in an organization which he understands', because it is his own. The important thing is not to make the native producer do something, but to make him want to do it. He does not like making experiments, and is easily discouraged when they are not a success; therefore he should only be taught methods which have been thoroughly tested.

There are other difficulties, such as the system of land tenure. Where this is communal, the individual will be less willing to invest capital in a permanent crop like oil-palms, and there is also natural indolence and carelessness to be overcome. To improve the economic activities of the native is a slow process-often too slow for the white man who wants to see quick results, but it is the safer way. The aim of colonial policy is not exploitation but development, and development within a primitive population cannot but be slow. It should also not be forgotten that a healthy development cannot have the increase of commercial production as its primary aim. It is far more important to raise the standard of life of the native, by helping him to grow sufficient food-crops of good quality for the maintenance of his own familyand to improve the general conditions of his village and home life; where that is done, increased wants will arise naturally and will lead to increased production.

\title{
Communautés indigènes traditionnelles et centres extra-coutumiers au Congo belge.
}

Dans un intéressant exposé de l'Agence Fides sur l'action catholique et sociale à Léopoldville, nous lisons que "la capitale du Congo belge possède depuis un an des corporations de métiers, groupant dans le cadre paroissial, sous la forme corporative, les employés, les serviteurs (boys), les maçons, les mécaniciens, les menuisiers, les artisans, les commerçants, les horticulteurs, les ouvriers. Cette dernière catégorie comprend les manœuvres et les ouvriers qui n'ont pas de travail déterminé. Pour nouer un lien entre les diverses corporations et leur millier d'adhérents, un nouveau bulletin a vu le jour, le Mangomba (Corporations). La nouvelle feuille paraîtra comme supplément à la Croix du Congo, quatre ou six fois par an. Elle donne des nouvelles des corporations, sert à la propagande parmi les indigènes et répond aux diverses interrogations que suscite le mouvement.'

Sans doute, pour aider les indigènes à atteindre un état politique, social et économique plus élevé il est de bonne méthode de maintenir et de développer les coutumes tribales, dans la mesure où elles peuvent coopérer au renouveau. Ce principe cependant, aussi fécond qu'il soit dans les communautés indigènes traditionnelles devient d'une application particulièrement complexe 
du moment qu'il s'agit des agglomérations artificielles, qui, à la suite de la mise en valeur du pays, sont nées à proximité des grands centres européens tels que Léopoldville, Elisabethville, Likasi, Coquilhatville, Stanleyville, Matadi, Albertville, Boma. Non seulement la grande diversité ethnique des individus qui les composent $y$ rend tout appel à un statut coutumier uniforme illusoire; des problèmes nouveaux s'y présentent auxquels la coutume ancestrale ne fournit pas de réponse. Ne pouvant cependant laisser ces agglomérations sans administration, le gouvernement les a constituées en centres indigènes extra-coutumiers. Ceci signifie qu'il entend substituer à la coutume un droit nouveau, qui s'inspirant de principes universellement humains sera assez souple cependant pour s'adapter à l'évolution économique et sociale de ces nouvelles collectivités.

En englobant dans des cadres administratifs la population amorphe d'une cité indigène, on ne restaure pas encore toutefois les liens spirituels qui dans l'ancienne organisation clanique ou tribale formaient toute la trame de la vie sociale. Il est extrèmement important de se rappeler ici qu'une fusion réellement organique du sens religieux et du sens social fut de tout temps à la base même de la société indigène. A l'encontre de celle-ci, les centres extracoutumiers, formés par une poussière d'individus déracinés, ignorent actuellement toute vie de relation tant dans le domaine religieux que dans l'ordre purement social. Ce ne sera là pourtant qu'un phénomène de courte durée. Le sens social du noir, qui trouve dans la solidarité clanique une expression aussi formelle, cherchera ici aussi à se manifester. En témoigne déjà la formation de certaines sociétés secrètes telles que le Tshimani. ${ }^{\text {I }}$

Reconnaissant cette tendance dont chaque indigène a le sentiment inné, les cuvres sociales de Léopoldville en ont fait un point de départ pour susciter des relations organiques nouvelles, adaptées aux temps nouveaux. Les corporations de métiers, substituant aux liens de parenté disparus des relations professionnelles, créent les cadres à l'intérieur desquels l'entreaide mutuelle peut à nouveau s'exercer. S'appuyant en outre sur une doctrine et une morale plus élevées, elles échappent au particularisme étroit que le culte des esprits conférait aux anciens groupements. Sous ce double aspect, l'expérience en cours à Léopoldville ouvre au développement du sens social chrétien des possibilités qu'il sera utile de suivre. (Communication du ProFESSEUR N. De CleENE.)

\section{'The Bush Speaks.'}

The purpose of this note is to draw attention to a remarkable book which, so far, is available only in a South African edition, and which may, there-

I E. De Jonghe, 'Formations récentes de sociétés secrètes au Congo belge, Africa, vol. ix, no. r, i936. 